Iranian Journal of Pathology | ISSN: 2345-3656

\title{
Evaluating of the Effects of Sub-MIC Concentrations of Gentamicin on Biofilm Formation in Clinical Isolates of Pseudomonas aeruginosa
}

\author{
Zahra Yousefpour ${ }^{1}$, Fateme Davarzani ${ }^{1}$, Parviz Owlia ${ }^{2 *}$ \\ 1. Department of Microbiology, Faculty of Medicine. Shahed University, Tehran, Iran \\ 2. Molecular Microbiology Research Center (MMRC), Faculty of Medicine, Shahed University, Tehran, Iran
}

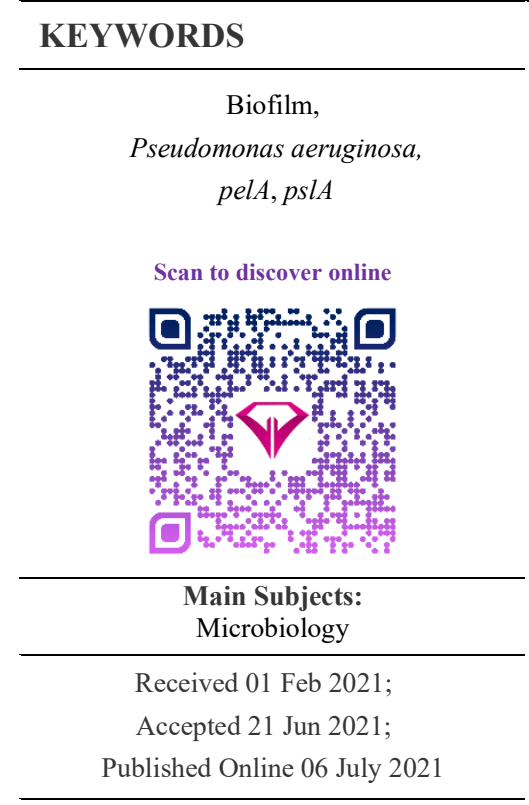

$\underline{10.30699 / I J P .20201 .524220 .2584 ~}$

\begin{abstract}
Background \& Objective: The ability of Pseudomonas aeruginosa to form biofilm has an important role in establishment of chronic phase of infections. Biofilm formation can be affected by antibiotics sub-MIC concentrations. The principal aim of the present study was to evaluate the effect of gentamicin at sub-MIC concentrations on biofilm formation in 100 Pseudomonas aeruginosa clinical isolates.
\end{abstract}

Methods: Determination of minimal inhibitory concentration of gentamicin for clinical isolates was done using micro broth dilution method. The amount of biofilm formation in the treated and untreated isolates with gentamicin sub-MIC (1/2\&1/4MIC) concentrations was evaluated using microtitre plate assay. pelA and $p s l A$ genes were detected in clinical isolates by PCR method.

Results: $99 \%$ of clinical isolates were biofilm producer. Different changes in amount of biofilm formation were observed in the treated clinical isolates with sub-MIC concentrations of gentamicin. Two dominant changes were observed in $80 \%$ of clinical isolates. These concentrations had inhibitory effect on biofilm formation in $46.4 \%$ of isolates and caused a significant decrease in its amount. While in $31.3 \%$ of the isolates, the biofilm formation was significantly increased. The frequency of pelA and pslA genes among clinical isolates was $100 \%$.

Conclusion: gentamicin sub-MIC concentrations cause different changes on biofilm formation of Pseudomonas aeruginosa clinical isolates. Therefore, further studies are needed for discovering new treatment strategies and using sub-MIC concentrations of the antibiotic in prevention and treatment of Pseudomonas aeruginosa infections.

\section{Corresponding Information: \\ Parviz Owlia, Molecular Microbiology Research Center (MMRC), Faculty of Medicine, Shahed University, Tehran, Iran E-mail: powlia@gmail.com}

Copyright $(C)$ 2021. This is an open-access article distributed under the terms of the Creative Commons Attribution- 4.0 International License which permits Share, copy and redistribution of the material in any medium or format or adapt, remix, transform, and build upon the material for any purpose, even commercially.

\section{Introduction}

Pseudomonas aeruginosa ( $P$. aeruginosa) is an opportunistic pathogen that can form biofilms and cause nosocomial infections, especially in immunocompromised individuals (1-3). P. aeruginosa is known as a significant bacterium due to the ability to grow in minimal conditions, the high spread in nature and the ability to form biofilms (4). Biofilm formation is the main strategy of Pseudomonas aeruginosa to survive in harsh environmental conditions $(5,6)$. In the chronic phase of infections, there is a decrease in activity of most virulence factors while biofilm formation acts as a virulence factor (7). Biofilm formation reduces the effect of antimicrobial agents (8). More than $80 \%$ of bacterial infections and about $65 \%$ of nosocomial infections are related to biofilm $(9$, $10)$. The biofilm is a complex microbial community besieged by a polysaccharide or protein matrix $(1,6)$. Extracellular polymeric substance (EPS) or exopoly- saccharide is a viscous organic substance which is essential for the formation of biofilm structure. It consists of polysaccharides, proteins and nucleic acids. Most polysaccharides produced by $P$. aeruginosa include psl, pel and alginate. pel is a glucose cationic exopolysa-ccharide that has a major role in the establishment of solid-surface biofilms. Non-mucoid strains use pel or psl polysaccharide as the primitive constructional scaffold. The pel has a protective role in the biofilm and increases resistance to antibiotics. The $p s l$ gene cluster is distributed around the cell surface and strengthens the structural scaffold and improves the establishment of microcolonies. It also plays a main role in the primary attachment of sessile cells to living and non-living surfaces. The main mechanism of resistance to antibiotics and other antimicrobial factors is associated with biofilms (1). Bacterial growth in biofilm can increase the MIC of antimicrobial agents 
100-1000 times (11). For a variety of reasons, the dose of antibiotic given to the patient may not achieve its optimal level (minimum inhibitory concentration, MIC or minimum bactericidal concentration, $\mathrm{MBC}$ ) at the affected tissue place, and the bacteria expose to subMIC concentrations (12). Therefore, bacteria are typically exposed to sub-MIC concentrations of antimicrobial factors (13). Sub-MIC concentrations of antibiotics alter the physiological and biochemical integrity of bacteria and impair some bacterial functions (12). It can also affect bacterial virulence agents such as adhesion, cell surface hydrophobicity, biofilm formation, sensitivity to oxidative stress and motility. According to recent studies, the utilization of sub-MIC concentrations of some antibiotics, such as macrolides, may inhibit biofilm formation and be useful in the treatment of $P$. aeruginosa infections (14). On the other hand, sub-MIC concentrations of antimicrobial agents can act as a stimulus for bacterial biofilm formation $(15,16)$. The effect of sub-MIC concentrations of aminoglycosides on biofilm formation has been investigated in the recent years. According to some studies, sub-MIC concentrations of aminoglycosides inhibit biofilm in $P$. aeruginosa (17). Hoffman et al. showed that sub-MIC concentrations of gentamicin stimulate biofilm formation, while the results of Hemati et al.'s study showed that sub-MIC concentrations of gentamicin have no stimulatory effect on biofilm formation $(13,18)$. Due to different results of studies, in this research, the effect of gentamicin 1/2 MIC and 1/4 MIC concentrations investigated on biofilm formation in $P$. aeruginosa clinical isolates was.

\section{Material and Methods}

\section{Microorganisms and Growth Conditions}

In this study, 100 clinical isolates of $P$. aeruginosa from different specimens (wound, urine, blood, etc.) were investigated. Samples collected from several selected hospitals in Tehran. Identification of clinical isolates was done by microbiological experiments including Gram staining, catalase and oxidase production, Oxidation-Fermentation (OF) test, culture on TSI medium and growth at $42^{\circ} \mathrm{C}$. For final confirmation of the isolates, the oprL gene, which is specific to $P$. aeruginosa, was amplified by PCR and traced by electrophoresis. Clinical isolates were kept at $-70^{\circ} \mathrm{C}$ in Nutrient broth (Merck, Germany) comprising $15 \%$ glycerol for use during the study. $P$. aeruginosa PAO1 standard strain was utilized as a positive control for biofilm assay.

\section{Molecular Detection of pelA, oprL and psl Genes}

PCR method was used for detecting pelA, pslA and oprL genes in $P$. aeruginosa. Specifications of primers are given in Table $1(19,20)$. The method is as follows: boiling method was used to extract the bacterial DNA; reaction materials (ddH2O: Primer R: $0.5 \mu \mathrm{L}$, Primer F: $0.5 \mu \mathrm{L}$, Taq DNA Polymerase Master Mix: $12.5 \mu \mathrm{L}$, Template DNA: $5 \mu \mathrm{L}, 6.5 \mu \mathrm{L}$ were transferred into microtubes, and for the oprL gene according to the protocol; Initial denaturation at $95^{\circ} \mathrm{C}$ for $5 \mathrm{~min}, 30$ cycles included; denaturation at $95^{\circ} \mathrm{C}$ for $30 \mathrm{~s}$, annealing at $57^{\circ} \mathrm{C}$ for $30 \mathrm{~s}$ and extension at $72^{\circ} \mathrm{C}$ for 1 min, and a final extension at $72^{\circ} \mathrm{C}$ for $10 \mathrm{~min}$ and for pelA and pslA genes according to the protocol; initial denaturation at $94^{\circ} \mathrm{C}$ for $5 \mathrm{~min}$, including 34 cycles; denaturation at $94^{\circ} \mathrm{C}$ for $30 \mathrm{~s}$, annealing at $59^{\circ} \mathrm{C}$ for 30 $\mathrm{s}$, and extension at $72^{\circ} \mathrm{C}$ for $1 \mathrm{~min}$ and a final extension at $72^{\circ} \mathrm{C}$ for $10 \mathrm{~min}$, it was placed in a thermocycler (BioRad-USA). Finally, PCR products were electrophoresed in $2 \%$ agarose gel which stained with safe stain (Sinacolon, Iran).

Table 1. Primer sequences of pelA, psl and oprL genes

\begin{tabular}{|c|c|c|c|}
\hline Gene & Primer sequence & Product length & Reference \\
\hline$p s l A$ & $\begin{array}{l}\text { Forward: 5'-TCCCTACCTCAGCAGCAAGC -3' } \\
\text { Reverse: 5'- TGTTGTAGCCGTAGCGTTTCTG -3' }\end{array}$ & $656 \mathrm{bp}$ & (20) \\
\hline pelA & $\begin{array}{c}\text { Forward: 5'-CATACCTTCAGCCATCCGTTCTTC-3' } \\
\text { Reverse: 5' - CGCATTCGCCGCACTCAG -3' }\end{array}$ & $786 \mathrm{bp}$ & (20) \\
\hline oprL & $\begin{array}{l}\text { Forward: 5'- ATGGAAATGCTGAAATTCGGC -3' } \\
\text { Reverse: 5' - CTTCTTCAGCTCGACGCGACG -3' }\end{array}$ & $504 \mathrm{bp}$ & (19) \\
\hline
\end{tabular}

\section{MIC Determination (Minimum Inhibitory Concentration)}

Minimal Inhibitory Concentration (MIC) of gentamicin for $P$. aeruginosa clinical isolates and standard strain PAO1 was determined by microbroth dilution method based on CLSI protocol (21). First, serial dilutions $(0.125-2048 \mu \mathrm{g} / \mathrm{mL})$ were prepared from gentamicin stock solution with an ultimate concentration of $5,120 \mu \mathrm{g} / \mathrm{mL}$ and transferred to microplate wells. Then Bacterial suspension equivalent to $0.5 \mathrm{McF}$ arland standard was added to the wells. Gentamicin-free bacterial culture was used as positive control and Mueller Hinton Broth (Merck, Germany) medium was used as a negative control and were incubated for $24 \mathrm{~h}$ at $37^{\circ} \mathrm{C}$. Then the minimum concentration of gentamicin, which inhibited bacterial growth, considered as MIC. Sensitive and resistant isolates according to CLSI (MIC $\leq 4 \mu \mathrm{g} / \mathrm{mL}$ : sensitive، $\mathrm{MIC} \geq 16 \mu \mathrm{g} / \mathrm{mL}$ : resistant) were identified. 


\section{Biofilm Formation}

To evaluate the biofilm formation, microtiter plate method was used, with some changes (22). The Bacteria were cultured in MHB medium and incubation of bacteria was performed for $24 \mathrm{~h}$ at $37^{\circ} \mathrm{C}$. Four tubes were assigned for each isolate. Then, in tube 1 , the culture was diluted at a ratio of 1:100 with fresh MHB medium to give an OD of $0.5 \mathrm{McF}$ arland. In tube 2 , the culture was diluted at a ratio of 1:50 with fresh MHB medium to give an OD 0.2. In tubes 3 and 4, the microbial suspension of tube 2 was mixed in equal proportions with $\mathrm{MIC}$ and $1 / 2 \mathrm{MIC}$ concentrations of gentamicin, respectively. Therefore, in tubes 3 and 4, in the final volume, both the concentration of antibiotics and the concentration of bacteria were halved. $200 \mu \mathrm{L}$ of the contents of each of tubes 1,3 and 4 were transferred to microplate wells and were incubated for $24 \mathrm{~h}$ at $37^{\circ} \mathrm{C}$. Uninoculated $\mathrm{MHB}$ medium was utilized as a negative control and $P$. aeruginosa PAO1 standard strain was utilized as a positive control. The medium inside the wells was drained and washed 3 times with PBS (phosphatebuffered saline). To stabilize the biofilm, $200 \mu \mathrm{L}$ of $95 \%$ ethanol was added to each well and then stained by adding $100 \mu \mathrm{L}$ of $1 \%$ crystal violet for $5 \mathrm{~min}$. To remove excess dye, washing of the wells was performed with running water. Then were placed at room temperature until dry. Finally, $150 \mu \mathrm{L}$ of $33 \%$ acetic acid was added to the wells. The Optical Density (OD) of the wells was measured at $490 \mathrm{~nm}$ by ELISA Reader (PerkinElmer, USA). The experiment was done in triplicate for all 3 suspensions (contents of tubes 1, 3 and 4) related to each isolation. The mean values of three wells related to the suspension of each of tube 1 , 3 and 4 were recorded as ODt and the mean OD of three wells related to the suspension of the control tube was recorded as ODc. The formed biofilm levels were determined according to the Table 2 (23).

\section{Statistical Analysis}

Data was analyzed by GraphPad Prism software version 8.0.2 using One-Way ANOVA test. P-values less than 0.05 were considered as statistically significant differences.

\section{Results}

\section{PCR Results}

The frequency of opr $L$ gene, which is specific to $P$. aeruginosa gene, as well as pelA and pslA genes that are involved in biofilm formation, was $100 \%$ in the studied isolates.

Results of Electrophoresis of PCR products of oprL, pelA and pslA genes are given in Figure 1.

Table 2. Interpretation of biofilm formation

\begin{tabular}{|c|c|}
\hline Interpretation & The amount of OD \\
\hline Lack of biofilm formation & $\mathrm{OD}_{\mathrm{t}} \leq \mathrm{OD}_{\mathrm{c}}$ \\
\hline Weak biofilm & $\mathrm{OD}_{\mathrm{c}}<\mathrm{OD}_{\mathrm{t}}<2 \mathrm{x}_{\mathrm{c}} \mathrm{OD}_{\mathrm{c}}$ \\
\hline Intermediate biofilm & $2 \mathrm{x}_{\mathrm{c}}<\mathrm{OD}_{\mathrm{t}}<4 \mathrm{x} \mathrm{OD}_{\mathrm{c}}$ \\
\hline Strong biofilm & $\mathrm{OD}_{\mathrm{t}} \geq 4 \mathrm{x} \mathrm{OD}_{\mathrm{c}}$ \\
\hline
\end{tabular}

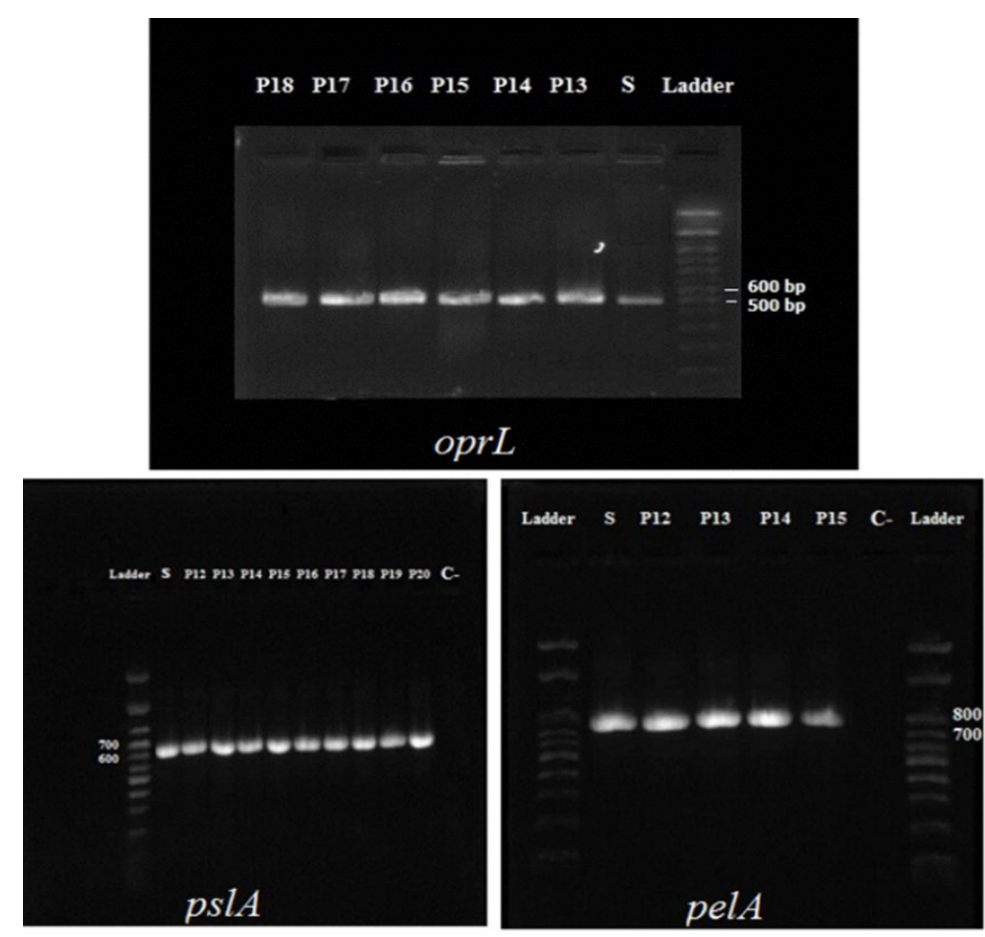

Fig. 1. Electrophoresis of PCR products of oprL (504bp), pelA (786bp), pslA (656bp) genes. P12- P20: clinical isolation. S: Positive control (PAO1). C-: Negative control (buffer and reagent water). 


\section{MIC Results}

MIC values of gentamicin for $P$. aeruginosa clinical isolates were evaluated according to CLSI tables. Accordingly, $71 \%$ of the strains were sensitive to gentamicin, and $29 \%$ were resistant. The frequency of MIC values of gentamicin is given in Table 3 .

Effect of Sub-MIC Concentrations of Gentamicin on Biofilm Formation

Overall, $99 \%$ of clinical isolates were biofilm producer. The amount of biofilm formation of $P$. aeruginosa PAO1 standard strain decreased significantly in both of $1 / 2 \mathrm{MIC}$ and $1 / 4 \mathrm{MIC}$ concentrations and formed a strong biofilm in the absence of gentamicin. Frequency of amounts of biofilm formed in clinical isolates of $P$. aeruginosa in the presence and absence of gentamicin are given in Figure 2. Sub-MIC concentrations of gentamicin had different effects on clinical isolates of $P$. aeruginosa (Figure 3). In exposure to both $1 / 2 \mathrm{MIC}$ and $1 / 4 \mathrm{MIC}$ concentrations of gentamicin, $46.4 \%$ of the isolates had a significant decrease, and $31.3 \%$ had a significant increase (Table 4).

Table 3. Frequency of gentamicin MIC values in clinical isolates of $P$. aeruginosa

\begin{tabular}{|c|c|c|c|c|c|c|c|c|c|c|}
\hline $\mathrm{MIC}(\boldsymbol{\mu g} / \mathrm{mL})$ & $\mathbf{0 . 2 5}$ & $\mathbf{0 . 5}$ & $\mathbf{1}$ & $\mathbf{2}$ & $\mathbf{1 6}$ & $\mathbf{3 2}$ & $\mathbf{6 4}$ & $\mathbf{1 2 8}$ & $\mathbf{2 5 6}$ & $\mathbf{5 1 2}$ \\
\hline Frequency & 11 & 37 & 20 & 3 & 1 & 4 & 1 & 5 & 12 & $\mathbf{6}$ \\
\hline
\end{tabular}

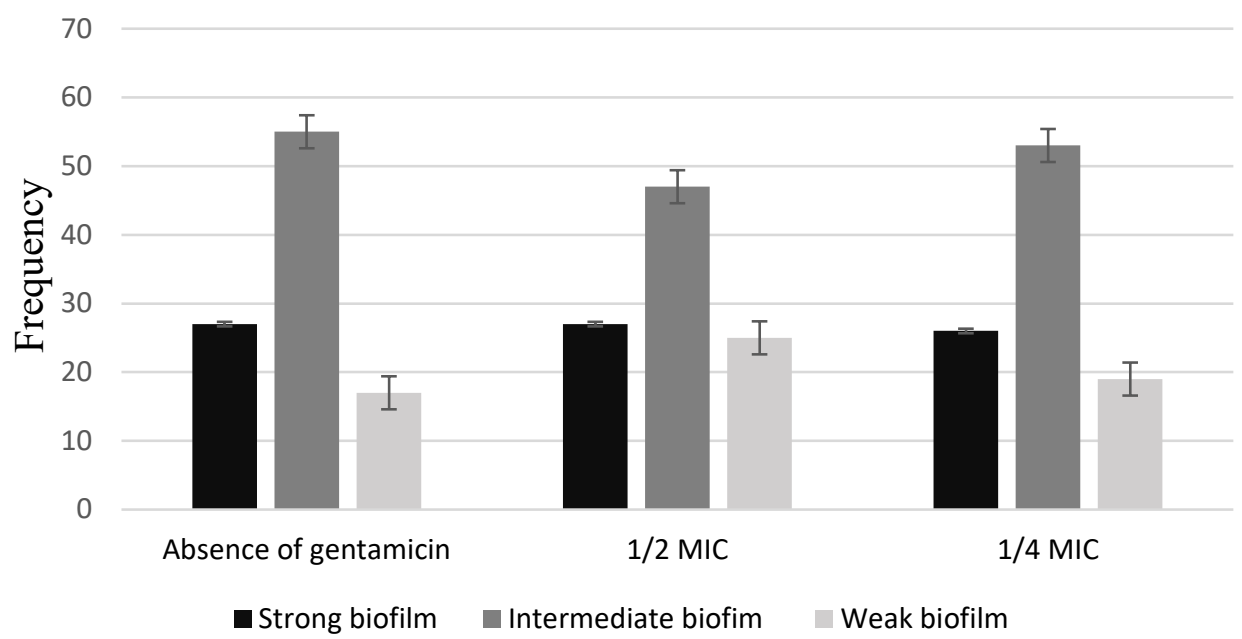

Fig. 2. Frequency of amounts of biofilm formed in clinical isolates of $P$. aeruginosa in the presence and absence of gentamicin

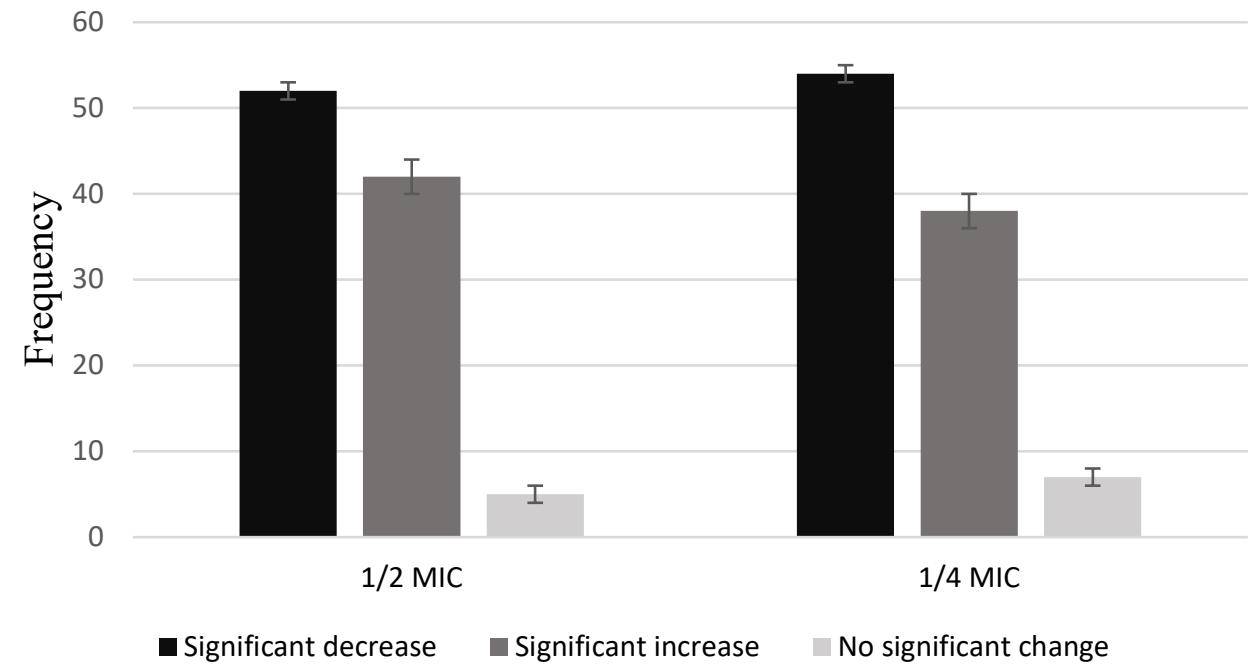

Fig 3. Frequency of changes in biofilm formation of clinical isolates of $P$. aeruginosa 
Table 4. Frequency of biofilm formation changes in clinical isolates of $P$. aeruginosa in the presence of $1 / 2$ MIC and $1 / 4$ MIC concentrations of gentamicin

\begin{tabular}{|c|c|c|}
\hline Type of changes & Frequency & Percentage \\
\hline Significant increase in 1/2 $\mathbf{M I C}$ and 1/4 MIC & 31 Isolates & $\% 31.3$ \\
\hline Significant decrease in 1/2 MIC and 1/4 MIC & 46 isolates & $\% 46.4$ \\
\hline Significant increase in 1/2 $\mathbf{M I C}$, Significant decrease in 1/4 MIC & 5 isolates & $\% 5$ \\
\hline Significant decrease in 1/2 MIC, significant increase in 1/4 MIC & 3 isolates & $\% 3$ \\
\hline Significant increase in 1/2 MIC, No Significant change in 1/4 MIC & 6 isolates & $\% 6$ \\
\hline No Significant change in 1/2 MIC, significant increase in 1/4 MIC & 4 isolates & $\% 4$ \\
\hline Significant decrease in 1/2 MIC, No Significant change in 1/4 MIC & 3 isolates & $\% 3$ \\
\hline No Significant change in $\mathbf{1 / 2}$ MIC and 1/4 MIC & 1 isolate & $\% 1$ \\
\hline
\end{tabular}

\section{Discussion}

$P$. aeruginosa is an opportunistic pathogen with minimal nutritional requirements and causes various types of nosocomial infections (24). This bacterium is able to form biofilms therefore often found in patients with chronic infections (25). Biofilm formation leads to the progression of the acute phase of infections to the chronic phase and plays a significant role in $P$. aeruginosa infection (26). Therefore, removal of bacteria and its biofilm is essential for the treatment of infections (27). Biofilms are resistant to antibiotics and cleared by the host immune system and are difficult to remove by antibiotics $(28,29)$. According to studies, treatment with sub-MIC concentrations of antimicrobial agents is effective on biofilm formation in $P$. aeruginosa (26). Sub-MIC concentrations of some antibiotics have been reported in a number of studies as inducer and in others as inhibitors of biofilm formation. It is essential that the effect of sub-MIC concentrations of antibiotics evaluated in different clinical isolates, as bacteria are subjected to sub-MIC concentrations of antibiotics at the beginning and end of the treatment regimen, between doses, or during continuous low-dose treatment (30). In our study, the biofilm formation of 100 clinical isolates of $P$. aeruginosa was investigated in the treated and untreated state with sub-MIC concentrations of gentamicin. According to Bahador et al. 98.6\% of clinical isolates of $P$. aeruginosa (75 isolates) from diverse samples, were able to form biofilms, which formed $60 \%$ of the isolates a strong biofilm, $34.3 \%$ a moderate biofilm and $4.3 \%$ a weak biofilm (31). Bahador et al. investigated the biofilm formation in antibiotic-untreated isolates, while we investigated the isolates in both of antibiotic-treated and untreated state. Based on the study of Purnajaf et al., of 143 clinical isolates of $P$. aeruginosa isolated from patients with cystic fibrosis, $78.2 \%$ of the isolates formed biofilm and pelA and pslA genes were present in 57.3\% and $89.5 \%$ of the isolates, sequentially (32). Ghadakaz et al. studied 104 clinical isolates of $P$. aeruginosa, and observed biofilm formation in $50.9 \%$ of the isolates, and the frequency of pelA and pslA genes were $45.2 \%$ and $83.7 \%$, respectively (20). Based on the results of our study, all isolates $(100 \%)$ were positive for pelA and $p s l A$ genes. Overall, $99 \%$ of the isolates formed the biofilm and only one isolate did not form a biofilm despite having the mentioned genes. Intermediate biofilm had the highest frequency in the amount of biofilm formed by isolates. So that in the absence of gentamicin and in exposure to $1 / 2 \mathrm{MIC}$ and 1/4 MIC concentration of gentamicin, 55, 47 and 53 isolates were formed intermediate biofilm, respectively. Variances in studied isolates caused differences in the results of mentioned studies compared to the results of present study. Various studies have reported different effects of antibiotics sub-MIC concentrations on biofilm formation and other factors related to bacterial pathogenesis. Otani et al. evaluated the expression of pelA and pslA genes and the biofilm formation of PAO1 strain in the untreated and treated state with ceftazidime sub-MIC concentrations (1/4, 1/8 and 1/16 MIC). In their study, sub-MIC concentrations of ceftazidime reduced the expression of pelA and pslA genes by 1.8- 2.1 times and also reduced the biofilm formation of $P A O 1$ strain (26). According to Bala et al. sub-MIC concentrations of azithromycin had an inhibitory effect on biofilm formation, production of signaling molecules of quorum sensing and motility of 25 clinical isolates of $P$. aeruginosa (28). The results of our study showed various changes in the biofilm formation of clinical isolates of $P$. aeruginosa in exposure to sub-MIC concentrations of gentamicin. The two main effects of these concent-rations were inhibitory effect and stimulatory effect on biofilm formation which were observed in $46.4 \%$ and $31.3 \%$ of isolates, respectively. The results of our study in PAO1 strain and $46.4 \%$ of the isolates are in accordance with Otani and Bala study. The results of a number of studies indicate the stimulatory effect of sub-MIC concentrations of antibiotics on the biofilm formation of various bacteria. R. Hoffman et al. obser-ved that tobramycin sub-MIC concentrations stimula-ted biofilm formation in $P$. aeruginosa PAO1 (18). This is against our results and the Otani's study on PAO1. It seems that the difference in the type of antibiotic used causes a difference in the results. Hemmati et al. observed that sub-MIC concentrations of ceftazidime, piperacillin, ticarcillin, carbonicillin, amikacin, 
ciprofloxacin, and the biocides, including chlorhexidine and benzalkonium chloride, increased the biofilm formation in 10 clinical isolates of $P$. aeruginosa (13). Based on the study of W. Garey et al. sub-MIC concentrations of clarithromycin increased the adhesion and volume of biofilm of 44 clinical isolates of $P$. aeruginosa (15). In the present study, in $31.3 \%$ of isolates, gentamicin sub-MIC concentrations increased biofilm formation. Majtan et al. found that the 1/4 MIC concentrations of the antibiotics gentamicin, amikacin, tobramycin, streptomycin, netilmicin, ciprofloxacin, norfloxacin, afloxacin and nalidixicacid, had an inhibitory effect on alginate (One of the important polysaccharides of biofilm structure in mucoid strains) production in one clinical strain of $P$. aeruginosa while 1/16 MIC concentration of some of these antibiotics had no effect on alginate and some others had a stimulatory effect on alginate production (33). We also observed that the reaction of some isolates in exposure to $1 / 2 \mathrm{MIC}$ concentration was different from their reaction to $1 / 4 \mathrm{MIC}$ concentration of gentamicin, in $5 \%$ of isolates. $1 / 2 \mathrm{MIC}$ concentration of gentamicin had a stimulatory effect and 1/4 MIC concentration of gentamicin had an inhibitory effect on biofilm formation. Reverse results were observed in $3 \%$ of isolates. On the other hand, in some other isolates, only one concentration affected the biofilm formation. Thus, in $6 \%$ of isolates, $1 / 2$ MIC concentration of gentamicin increased biofilm formation, but $1 / 4$ MIC concentration had no significant effect on biofilm formation. In $4 \%$ of isolates, the 1/4 MIC concentration of gentamicin induced biofilm formation, while the $1 / 2$ MIC concentration had no significant effect on biofilm formation. To confirm the different effects, the experiments were repeated and the same results were obtained again. Bruchmann et al. evaluated the effect of sub-MIC concentrations of several antibiotics on $P$. aeruginosa isolates collected from municipal wastewater. An increase was observed in biofilm biomass and thickness in treated state with sub-MIC concentration of sulfamethoxazole and erythromycin, in expression of resistance genes and efflux pump in the presence of sub-MIC concentrations of sulfonamides and in expression of quorum sensing genes in exposure to sub-MIC concentrations of macrolide. Sensitive isolates to these antibiotics had higher transcriptional activity (34). In the present study, the dominant change observed in both susceptible and gentamicin-resistant isolates included a reduction in biofilm formation. The inhibitory effect of sub-MIC concentrations of gentamicin was observed in $52.11 \%$ of susceptible isolates and $42.85 \%$ of resistant isolates. Based on the results of the studies, the response of bacteria to sub-MIC concentrations of antibiotics depends on the type of antibiotic, type of isolate or strain and test conditions (34). Because subMIC concentrations of antibiotics play a significant role in the regulation and modification of a wide range of bacterial genes, including virulence genes, and given that the expression of virulence genes and biofilm formation in $P$. aeruginosa is regulated by the quorum sensing, the changes observed in exposure to sub-MIC concentrations of antibiotics may be due to interaction with the quorum sensing system $(32,35)$.

\section{Conclusion}

The results of present study demonstrated two dominant reactions. The stimulating effect of sub-MIC concentrations of gentamicin on biofilm formation can make difficult the treatment and lead to the creation or persistence of chronic infections. The inhibitory effect of these concentrations could provide new therapeutic strategies. Due to the different results of the present and other studies, the use of sub-MIC concentrations in the treatment process requires more comprehensive studies. It seems that the study of factors involved in the different responses of bacteria to sub-MIC concentrations of antibiotics can be helpful in achieving the desired results.

\section{Acknowledgements}

None.

\section{Conflict of Interest}

None.

\section{References}

1. Skariyachan S, Sridhar VS, Packirisamy S, Kumargowda ST, Challapilli SB. Recent perspectives on the molecular basis of biofilm formation by Pseudomonas aeruginosa and approaches for treatment and biofilm dispersal. Folia Microbiol (Praha). 2018;63(4):413-32. [DOI:10.1007/s12223-018-0585-4] [PMID]

2. Kazemi Moghaddam E, Owlia P, Jahangiri A, Rasooli I, Rahbar MR, Aghajani M. Conserved OprF as a Selective Immunogen against Pseudomonas aeruginosa. Iran J Pathol.

\section{7;12(2):165-70. [DOI:10.30699/ijp.2017.24875] [PMCID]}

3. Hoseinpoor Mohammad Abadi Z, Mokhtari A, Ebrahimi Kahrizsangi A. Evaluation of the Effect of a Number of Commercial Disinfectants on Pseudomonas aeruginosa Isolates Obtained from Human Infection Cases. Iranian Journal of Medical Microbiology. 2020;14(2):138-53. [DOI:10.30699/ijmm.14.2.138] 
4. Erdal B, Yalinay M, Elmas C, Yazici GN. [Investigation of Pseudomonas aeruginosa Biofilm Formation and Quorum Sensing Genes in Piperacillin/Tazobactam and Ciprofloxacin Subminimal Inhibitory Concentrations]. Mikrobiyol Bul. 2020;54(4):547-58 [DOI:10.5578/mb.70087] [PMID]

5. Hadadi-Fishani M, Khaledi A, Fatemi-Nasab ZS Correlation between biofilm formation and antibiotic resistance in Pseudomonas aeruginosa: a meta-analysis. Infez Med. 2020;28(1):47-54.

6. Thi MTT, Wibowo D, Rehm BHA. Pseudomonas aeruginosa Biofilms. Int J Mol Sci. 2020;21(22):8671. [DOI:10.3390/ijms21228671] [PMID] [PMCID]

7. Shi N, Gao Y, Yin D, Song Y, Kang J, Li X, et al. The effect of the sub-minimal inhibitory concentration and the concentrations within resistant mutation window of ciprofloxacin on MIC, swimming motility and biofilm formation of Pseudomonas aeruginosa. Microb Pathog. 2019; $137: 103765$.

[DOI:10.1016/j.micpath.2019.103765] [PMID]

8. Azimi S, Kafil HS, Baghi HB, Shokrian S, Najaf K, Asgharzadeh M, et al. Presence of exoY, exoS, exoU and exoT genes, antibiotic resistance and biofilm production among Pseudomonas aeruginosa isolates in Northwest Iran. GMS Hyg Infect Control. 2016;11:Doc04.

9. Ochoa SA, Cruz-Cordova A, Rodea GE, CazaresDominguez V, Escalona G, Arellano-Galindo J, et al. Phenotypic characterization of multidrugresistant Pseudomonas aeruginosa strains isolated from pediatric patients associated to biofilm formation. Microbiol Res. 2015;172:68-78. [DOI:10.1016/j.micres.2014.11.005] [PMID]

10. Lee K, Yoon SS. Pseudomonas aeruginosa Biofilm, a Programmed Bacterial Life for Fitness. J Microbiol Biotechnol. 2017;27(6):1053-64. [DOI:10.4014/jmb.1611.11056] [PMID]

11. Bagge N, Schuster M, Hentzer M, Ciofu O, Givskov M, Greenberg EP, et al. Pseudomonas aeruginosa biofilms exposed to imipenem exhibit changes in global gene expression and betalactamase and alginate production. Antimicrob Agents Chemother. 2004;48(4):1175-87. [DOI:10.1128/AAC.48.4.1175-1187.2004] [PMID] [PMCID]

12. Bahram A, Baghchesaraie H, Faghihi MHO. Effect of different sub MIC concentrations of penicillin, vancomycin and ceftazidime on morphology and some biochemical properties of Staphylococcus aureus and Pseudomonas aeruginosa isolates. Iranian Journal of Microbiology. 1970;1(1).
13. Hemati S, Sadeghifard N, Ghafurian S, Maleki F, Mahdavi Z, Hassanvand A, et al. The association of biofilm formation and sub-minimal inhibitory concentrations of antimicrobial agents. 2016.

14. Roudashti S, Zeighami H, Mirshahabi H, Bahari S, Soltani A, Haghi F. Synergistic activity of subinhibitory concentrations of curcumin with ceftazidime and ciprofloxacin against Pseudomonas aeruginosa quorum sensing related genes and virulence traits. World J Microbiol Biotechnol. 2017;33(3):50. [DOI:10.1007/s11274-016-2195-0] [PMID]

15. Garey KW, Vo QP, Lewis RE, Saengcharoen W, LaRocco MT, Tam VH. Increased bacterial adherence and biomass in Pseudomonas aeruginosa bacteria exposed to clarithromycin. Diagn Microbiol Infect Dis. 2009;63(1):81-6. [DOI:10.1016/j.diagmicrobio.2008.09.007] [PMID]

16. Hemati S, Kouhsari E, Sadeghifard N, Maleki A, Omidi N, Mahdavi Z, et al. Sub-minimum inhibitory concentrations of biocides induced biofilm formation in Pseudomonas aeruginosa. New Microbes New Infect. 2020;38:100794. [DOI:10.1016/i.nmni.2020.100794] [ [PMCID]

17. Khan F, Lee JW, Javaid A, Park SK, Kim YM. Inhibition of biofilm and virulence properties of Pseudomonas aeruginosa by sub-inhibitory concentrations of aminoglycosides. Microb Pathog. 2020;146:104249. [DOI:10.1016/j.micpath.2020.104249] [PMID]

18. Hoffman LR, D'Argenio DA, MacCoss MJ, Zhang Z, Jones RA, Miller SI. Aminoglycoside antibiotics induce bacterial biofilm formation. Nature. 2005;436(7054):1171-5. [DOI:10.1038/nature03912] [PMID]

19. Owlia P, Nosrati R, Alaghehbandan R, Lari AR. Antimicrobial susceptibility differences among mucoid and non-mucoid Pseudomonas aeruginosa isolates. GMS Hyg Infect Control. 2014;9(2):Doc13.

20. Ghadaksaz A, Fooladi AAI, Mahmoodzadeh Hosseini H, Amin M. The prevalence of some Pseudomonas virulence genes related to biofilm formation and alginate production among clinical isolates. Journal of Applied Biomedicine. 2015;13(1):61-8.

[DOI:10.1016/j.jab.2014.05.002]

21. CLSI. Clinical and Laboratory Standards Institute. Performance Standards for Antimicrobial Susceptibility Testing; 21st Informational Supplement. M100-S21. CLSI Wayne, PA; 2011. 
22. Zmantar T, Kouidhi B, Miladi H, Mahdouani K, Bakhrouf A. A microtiter plate assay for Staphylococcus aureus biofilm quantification at various $\mathrm{pH}$ levels and hydrogen peroxide supplementation. New Microbiol. 2010;33(2):137-45.

23. Saidi N, Owlia P, Marashi SMA, Saderi H. Inhibitory effect of probiotic yeast Saccharomyces cerevisiae on biofilm formation and expression of alpha-hemolysin and enterotoxin A genes of Staphylococcus aureus. Iran J Microbiol. 2019;11(3):246-54. [DOI:10.18502/ijm.v11i3.1331] [PMID] [PMCID]

24. Harjai K, Khandwahaa RK, Mittal R, Yadav V, Gupta V, Sharma S. Effect of $\mathrm{pH}$ on production of virulence factors by biofilm cells of Pseudomonas aeruginosa. Folia Microbiol (Praha). 2005;50(2):99-102.

[DOI:10.1007/BF02931455] [PMID]

25. van 't Wout EF, van Schadewijk A, van Boxtel R, Dalton LE, Clarke HJ, Tommassen J, et al. Virulence Factors of Pseudomonas aeruginosa Induce Both the Unfolded Protein and Integrated Stress Responses in Airway Epithelial Cells. PLoS Pathog. 2015;11(6):e1004946. [DOI:10.1371/journal.ppat.1004946] [ $\underline{\text { PMID] }}$ [PMCID]

26. Otani S, Hiramatsu K, Hashinaga K, Komiya K, Umeki K, Kishi K, et al. Sub-minimum inhibitory concentrations of ceftazidime inhibit Pseudomonas aeruginosa biofilm formation. J Infect Chemother. 2018;24(6):428-33. [DOI:10.1016/j.jiac.2018.01.007] [PMID]

27. Ghadam P, Akhlaghi F, Ali AA. One-step purification and characterization of alginate lyase from a clinical Pseudomonas aeruginosa with destructive activity on bacterial biofilm. Iran $\mathrm{J}$ Basic Med Sci. 2017;20(5):467-73.

28. Bala A, Kumar R, Harjai K. Inhibition of quorum sensing in Pseudomonas aeruginosa by azithromycin and its effectiveness in urinary tract infections. J Med Microbiol. 2011;60(Pt 3):3006. [DOI:10.1099/jmm.0.025387-0] [PMID]
29. Wannigama DL, Hurst C, Hongsing P, Pearson L, Saethang T, Chantaravisoot N, et al. A rapid and simple method for routine determination of antibiotic sensitivity to biofilm populations of Pseudomonas aeruginosa. Ann Clin Microbiol Antimicrob. 2020;19(1):8. [DOI:10.1186/s12941-020-00350-6] [PMID] [PMCID]

30. Kaplan JB. Antibiotic-induced biofilm formation. Int J Artif Organs. 2011;34(9):737-51. [DOI:10.5301/ijao.5000027] [PMID]

31. Bahador N, Shoja S, Faridi F, DozandehMobarrez B, Qeshmi FI, Javadpour S, et al. Molecular detection of virulence factors and biofilm formation in Pseudomonas aeruginosa obtained from different clinical specimens in Bandar Abbas. Iran J Microbiol. 2019;11(1):2530. [DOI:10.18502/ijm.v11i1.701] [PMID] [PMCID]

32. Pournajaf A, Razavi S, Irajian G, Ardebili A, Erfani $\mathrm{Y}$, Solgi $\mathrm{S}$, et al. Integron types, antimicrobial resistance genes, virulence gene profile, alginate production and biofilm formation in Iranian cystic fibrosis Pseudomonas aeruginosa isolates. Infez Med. 2018;26(3):226-36.

33. Majtan V, Hybenova D. Inhibition of Pseudomonas aeruginosa alginate expression by subinhibitory concentrations of antibiotics. Folia Microbiol (Praha). 1996;41(1):61-4. [DOI:10.1007/BF02816342] [PMID]

34. Bruchmann J, Kirchen S, Schwartz T. Subinhibitory concentrations of antibiotics and wastewater influencing biofilm formation and gene expression of multi-resistant Pseudomonas aeruginosa wastewater isolates. Environ Sci Pollut Res Int. 2013;20(6):3539-49. [DOI:10.1007/s11356-013-1521-4] [PMID]

35. Linares JF, Gustafsson I, Baquero F, Martinez JL. Antibiotics as intermicrobial signaling agents instead of weapons. Proc Natl Acad Sci U S A. 2006;103(51):19484-9.

[DOI:10.1073/pnas.0608949103] [PMID] [PMCID] 